\title{
3D Computational Fluid Dynamic Modelling for Pulsatile Blood Wave Propagation in the Event of Car Crash
}

\author{
Jiling Feng*, Meiwen An ${ }^{* *}$, Sravanthi Sashikumar*, Weiyi Chen ${ }^{* *}$ \\ * School of Engineering, Manchester Metropolitan University, M1 5GD, UK \\ ** Institute of the Applied Mechanics and Biomedical Engineering, Taiyuan University of Technology, 030024, P.R China
}

\begin{abstract}
Blunt traumatic aortic rupture (BTAR) is one of the leading causes of rapid fatality in motor vehicle crashes. The mechanism of BTAR, however, is still not clear due to its complicated process. This paper looks the pattern alteration of blood wave propagation of the aorta caused by impact loading to identify the sources of rupture of aorta. In this paper, a 3D computational fluid dynamic (CFD) human aortic model was established. Pulsatile pressure and velocity, representing the cardiac transient pressure and velocity for the healthy adult, were applied at the inlet and outlets of aortic model as the boundary conditions. Blood flow propagation along the ascending aorta to thoracic descending aorta were analysed using ABAQUS CFD. The results indicate that the waves as a result of the impact loading has a significant effect on the patterns of blood wave propagation, which may be considered as one of the sources of rupture of aorta.
\end{abstract}

KEY WORDS: Blunt Traumatic Aortic Rupture, Blood Wave Propagation

\section{INTRODUCTION}

Blunt Traumatic Aortic Rupture (BTAR) refers to the tearing or rupture of the aorta wall during the car crash. It is one of the leading causes for rapid fatality in motor vehicle crashes. BTAR accounts for $21.4 \%$ of all deaths from motor vehicle accidents and approximately $80-85 \%$ of victims of BTAR die at the scene or on ways to hospitals ${ }^{[1,2]}$. Fig.1 shows that the distribution of BTAR victims as the function of the injury sites and impact types ${ }^{[1]}$. The findings from this study demonstrated that BTAR most often occurred in the descending aorta at the isthmus, which is referred as the 'classical' site of BTAR.

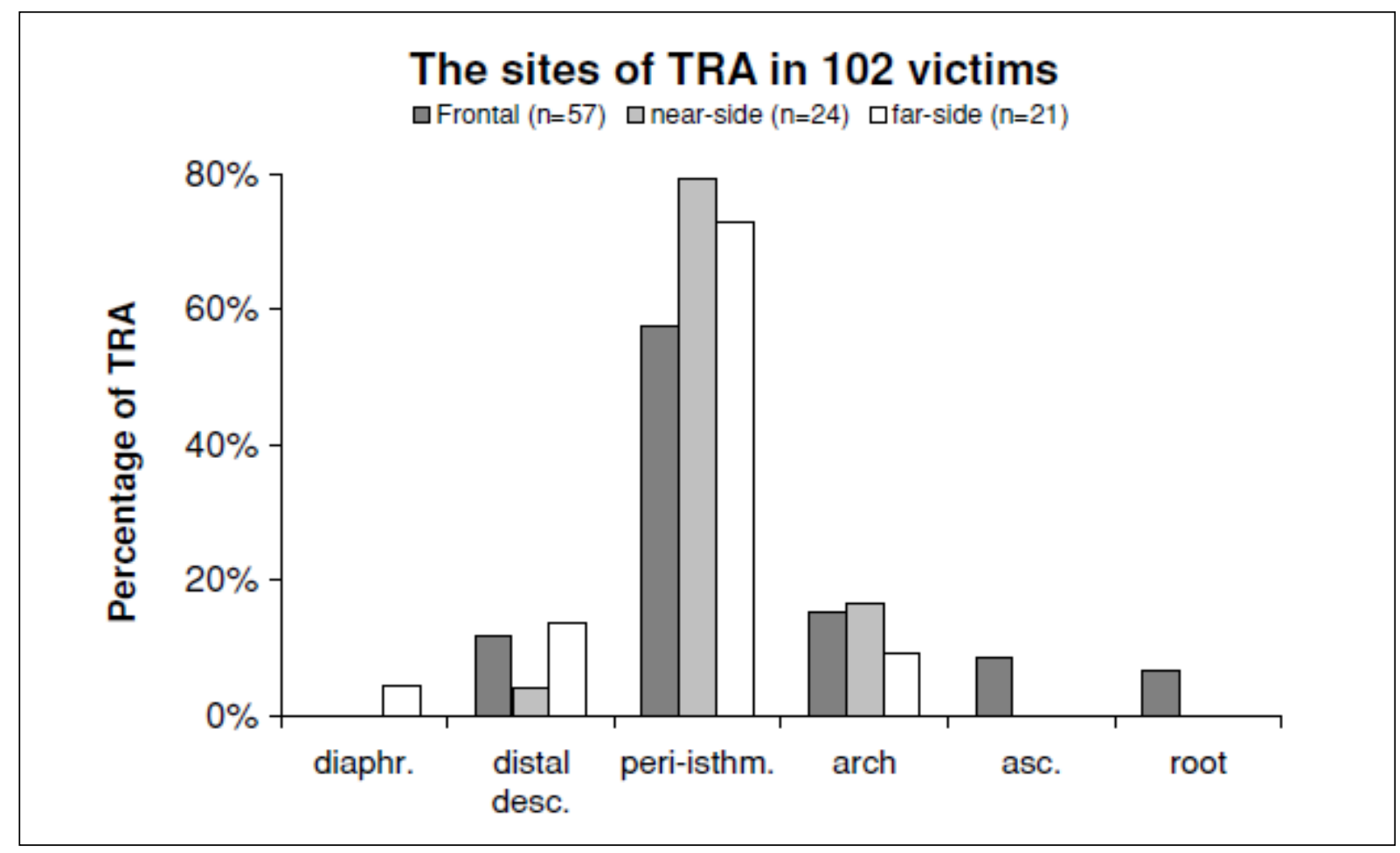

Fig. 1. Distribution function of the TRA (Traumatic rupture of aorta) victims in relation to the sites of injury and impact types ${ }^{[1]}$.

(Horizontal axis represents the sites of injury in aorta in abbreviations: 'diaphr.'--diaphragm; 'distal desc.' --distal descending aorta; 'peri-isthm.'--peri-isthmic aorta; and 'asc.'--ascending aorta) 
Apart from the automotive accidents, there are additional circumstances under which the aortic rupture may occur. BTAR fatalities were also found in motorcyclist crash, falling down from scaffolding and high work site, etc. It was reported that $93.3 \%$ of the overall mortality or $80 \%$ of on-site mortality of fatal accident can be attributed to $\mathrm{BTAR}^{[3]}$. In fact, the mortality rates as the result of BTAR are dramatically high, no matter whatever circumstances under which this kind of injury occurs.

To minimize the occurrence of aortic and other injuries due to car accident, significant efforts have been made in car design (include seat belts and airbags) over the past decades. The analysis by the National Highway Transportation Safety Administration (NHTSA) found that car seat belt have saved 168,524 US lives since 1960, and front airbags have saved 12,074 US lives ${ }^{[4]}$. The review report by Schulman, however, revealed that during 1993-2004, the overall incidence of blunt aortic injury that is associated with fatal vehicular crashes has remained high ${ }^{[5]}$. More recently, Sastry, based on UK Cooperative Crash Injury Study (CCIS) data during 1998-2007, found low-impact scenarios may account for two-thirds of blunt traumatic aortic rupture ${ }^{[6]}$. Sastry also found low-impact blunt traumatic aortic rupture is more commonly found at the old vehicle occupant. This finding is consistent with the finding by Welsh, where the body region most prone to injury for elderly vehicle occupant is chest ${ }^{[7]}$. It is understood that the lack of understanding of the BTAR mechanisms could mean that the overall fatality rate of blunt aortic injury during vehicle crash will remain high in the future, even in the presence of extensive use of safety seat belts and airbags.

In order to reduce the fatality ratio caused by the aortic rupture, the BTAR mechanisms have been investigated in a number of ways. Lundevall suggested that the injury of aorta is mainly caused by the sudden stretching of aorta ${ }^{[8]}$. Kivity thought that the significant pressure pulse increase due to the sudden occlusion of blood vessel wall in the aorta is key factor of BTAR ${ }^{[9]}$. Kivity indicated that shock waves, formed in the arterial system, could led to the rupture of aortas eventually. Since the aorta is believed to be entrapped between the anterior thoracic bony structure and the vertebral column during vehicle impact, a recent study suggested that it is the combined bending and shear stresses, induced by the entrapment, make a great contribution to the rupture of aorta ${ }^{[10-12]}$.

Literature review mentioned above revealed that the mechanism of BTAR is not clear in spite of BTAR been investigated widely to date. The main challenges are: (1) The mechanisms suggested by the different researchers are contradict to each other, where each investigation only focused on the individual factor, therefore, the systematical understanding of the mechanisms of BTAR is very limited; (2) It is hypothesized that pattern of blood wave propagation in the arterial system during car crash will be affected, however, investigation of the patterns of blood wave propagation under the impact loading is lacking. This paper, therefore, will focus on the blood wave propagation in the aorta under the impact loading and find out whether the alteration of patterns of the blood wave propagation is associated with the rupture of aorta.

The blood flow performance and the blood wave propagation in the aorta during car crash are investigated using computational modelling approach in this paper. A 3D computational fluid dynamic (CFD) model, representing the inside fluid domain of the adult aorta (aortic lumen), had been developed by using the ABAQUS CFD software. Pulsatile blood pressure and flow waveform will be used as the boundary conditions. The blood flow performance and the patterns of blood wave propagation in the normal conditions will be compared with those when the aorta is subjected to impact loading.

\section{METHODOLOGY}

In this paper, a computational fluid dynamic (CFD) model, representing the inside fluid domain of the adult aorta (aortic lumen) from ascending aorta to descending thoracic aorta has been developed. ABAQUS CFD software is used to simulate the blood flow behaviour during car crash.

In this model, the three major aortic branches including brachiocephalic artery, left carotid artery and left subclavian artery in the aortic arch were also included. The geometric and material properties of 

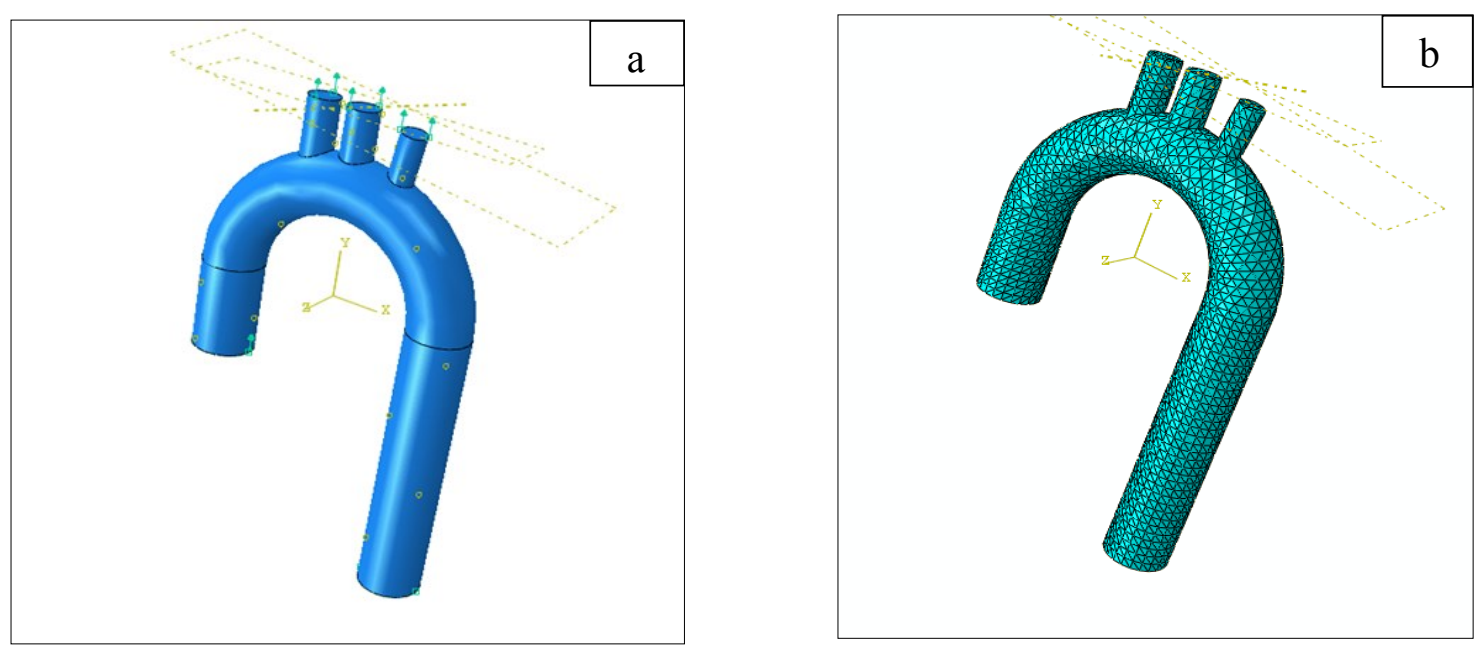

Fig. 2 (a) for geometric aortic model and the boundary conditions; (b) for aortic mesh model.

the aortic model are referred from the Voges's study for adult ${ }^{[13]}$. The model is consisted with six sections: namely, ascending aorta, aortic arch, descending aorta, and three branches (Fig.2a). The density $\left(1005 \mathrm{~kg} / \mathrm{m}^{3}\right)$ and viscosity $\left(3.2 \times 10^{-3}\right.$ Pa.s $)$ of blood have been assumed. The computational model is consisted by 20727 tetrahedral elements, shown in Fig.2b. Three types of boundary conditions have been applied to the inlet of ascending aorta, outlet of descending aorta and the outlet of branches in the aortic arch, respectively. The first type of boundary conditions were used to simulate the normal physiological conditions, where the pulsatile pressure waveform (Fig.3) was applied at inlet of the ascending of aorta and velocity waveform (Fig. 4, Fig. 5) was applied at outlet of descending aorta and three branches. The second type of boundary conditions were used to simulate the blood flow behaviours when the aorta is occluded at the outlet, therefore, zero velocity was applied. The third types of boundary conditions were used to simulate the blood flow behaviours when the aorta is subjected to the occlusion and the impact loading. At this situation, the descending aorta was fully blocked. Meanwhile, at the location of occlusion, an impulse signal, representing the addition pressure waveforms, generated by impact loading, was also applied as the boundary conditions. The blood vessel walls for all of the aortic sections were assumed rigid and no slip conditions were applied to the blood vessel walls.

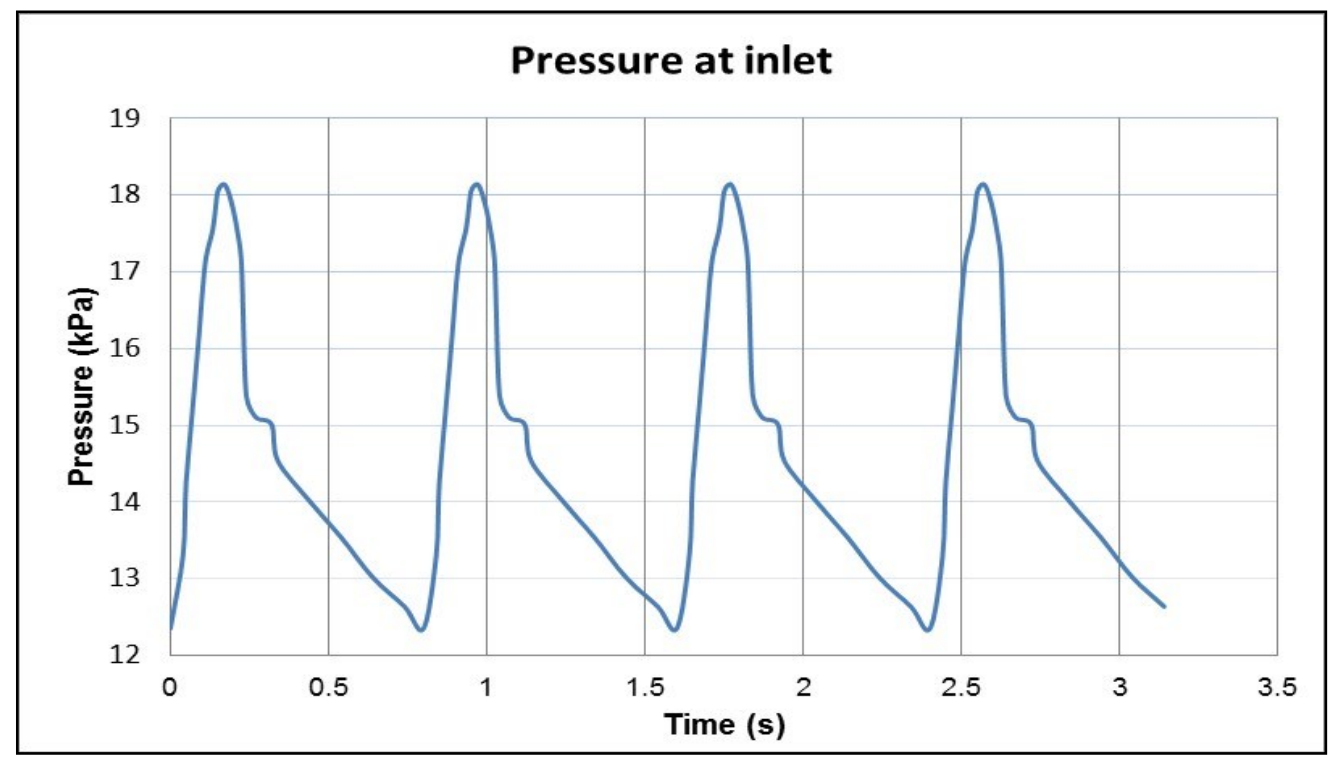

Fig. 3. Boundary conditions of the pulsatile blood pressure were applied at the inlet of ascending aorta 


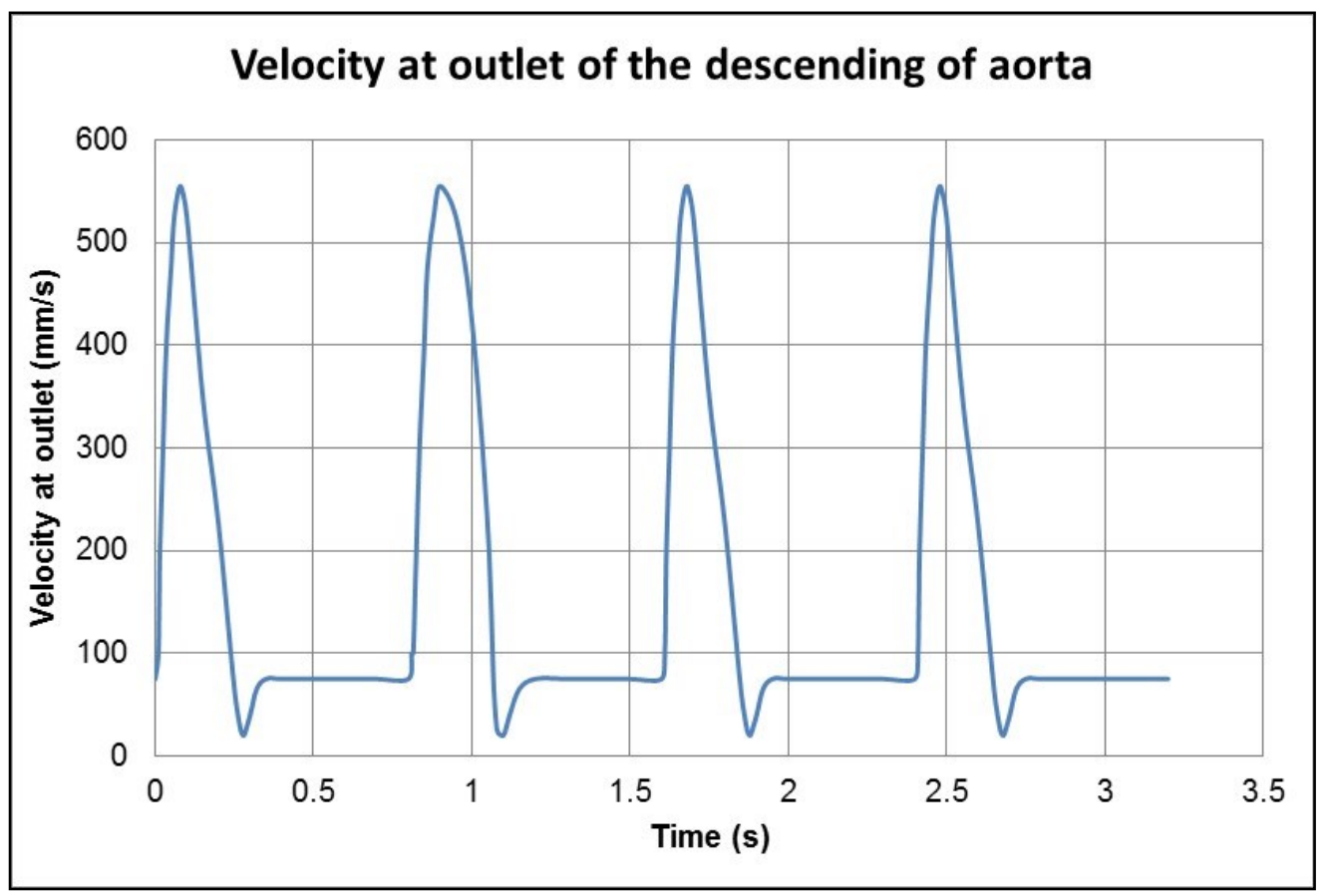

Fig. 4. Boundary conditions of the pulsatile blood velocity at the outlet of descending aorta

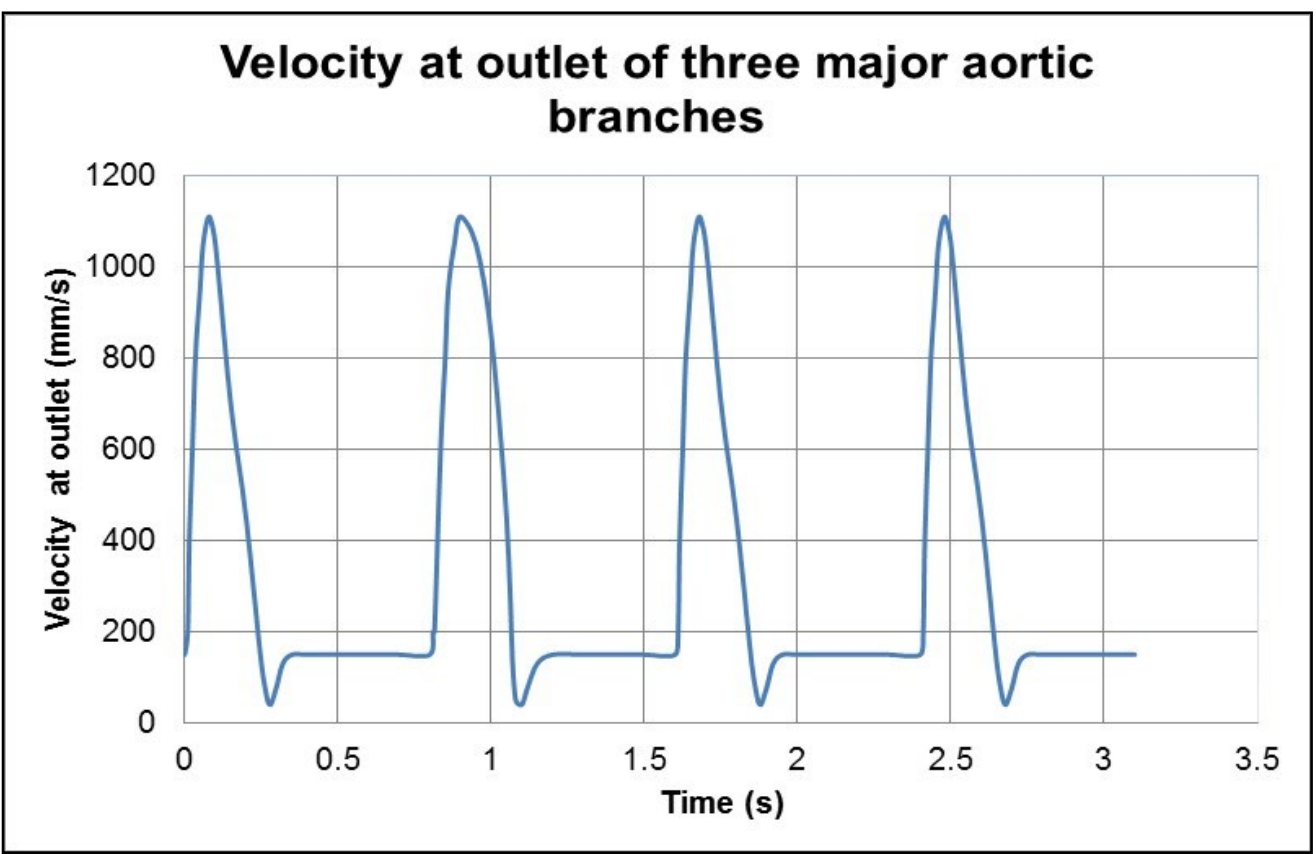

Fig. 5 Boundary conditions of the pulsatile blood velocity at the outlet of three major aortic branches

The pulsatile blood flow through 3D CFD aortic model was simulated with the incompressible NavierStokes equations. The governing equations, combined with the boundary conditions, were solved with the finite element method (FEM) based on the commercial software ABAQUS CFD. Since the rigid vessel wall is assumed in this study, the coupling of the deformation of the vessel wall and blood flow behaviour is not considered. The pressure and velocity waveform at several locations will be extracted. The pressure and velocity contour will also be presented so that the detailed pressure distribution and velocity profile along longitudinal symmetrical sections can be investigated. 


\section{RESULTS:}

The pressure distribution and flow profile under the three types of the boundary conditions were presented as the following:

\section{Normal physiological conditions}

The blood flow patterns at normal physiological condition are studied using 4 continuity stable cycles (Fig. 3, Fig. 4 \& Fig. 5). The pulsatile pressure and velocity waveforms were applied at the inlet and at the outlet. The results at the $4^{\text {th }}$ cycle are extracted and the pressure distributions at 4 key time points: $\mathrm{t}_{1}=2.404 \mathrm{~s}, \mathrm{t}_{2}=2.568 \mathrm{~s}, \mathrm{t}_{3}=2.693 \mathrm{~s}$ and $\mathrm{t}_{4}=3.168 \mathrm{~s}($ Fig. 6) are presented in Fig. 7.

As you can see from Fig. 7, at $\mathrm{t}_{1}=2.404 \mathrm{~s}$ when a cardiac cycle starts, the pressure distributions from the ascending aorta to descending aorta ranged from $12.89 \mathrm{kPa}$ to $12.44 \mathrm{kPa}$. It is the pressure difference between the ascending aorta and the descending aorta driving the flow from the upstream of aorta to the downstream. At peak of the systolic cardiac cycle $\left(\mathrm{t}_{2}=2.568 \mathrm{~s}\right)$, the pressure distribution ranges from $17.68 \mathrm{kPa}$ to $18.14 \mathrm{kPa}$, where the maximum pressure occurs at the outlet of descending aorta, indicating the blood flow may move backward to upstream at that moment. At $\mathrm{t}_{3}=2.693 \mathrm{~s}$ and $\mathrm{t}_{4}=3.168 \mathrm{~s}$, the pressures are distributed nearly uniform with the slight gradient between ascending aorta and descending aorta, which keeps the blood flow moving toward the peripheral arteries.

In addition, the pressure and velocity waveforms at 4 key locations (inlet of ascending of aorta, beginning of aortic arch, end of aortic arch and outlet of aorta) are presented in the Fig. 8. It was observed that pressure waveforms are similar in shape and the systolic peak gradually increase when the blood flow moving from ascending aorta to descending aorta.

\section{Blood flow behaviours when the aorta is occluded}

During car crash, it is supposed that occlusion occurs due to the impact loading acted on the human body. The occlusion would cause the blood flow waveforms to be reflected and moving towards the upstream of aorta, which may result in the rapid and significant increase of the pressure. The pressure waveforms at the outlet of descending aorta between the condition when the aorta is occluded and normal physiological conditions was compared (Fig. 9).

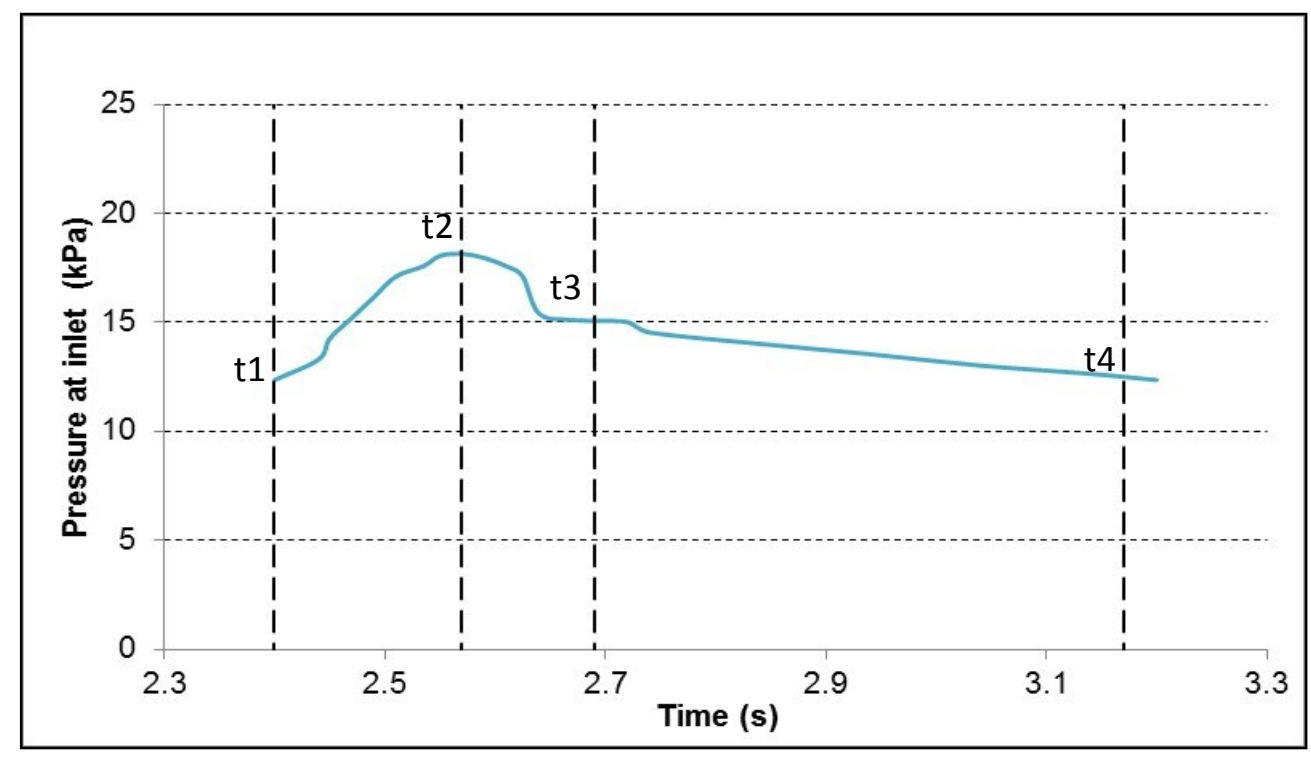

Fig. 6: Four key time points indicate the beginning of systolic phase, peak of systolic phase, midway and end of diastolic phase, respectively. 

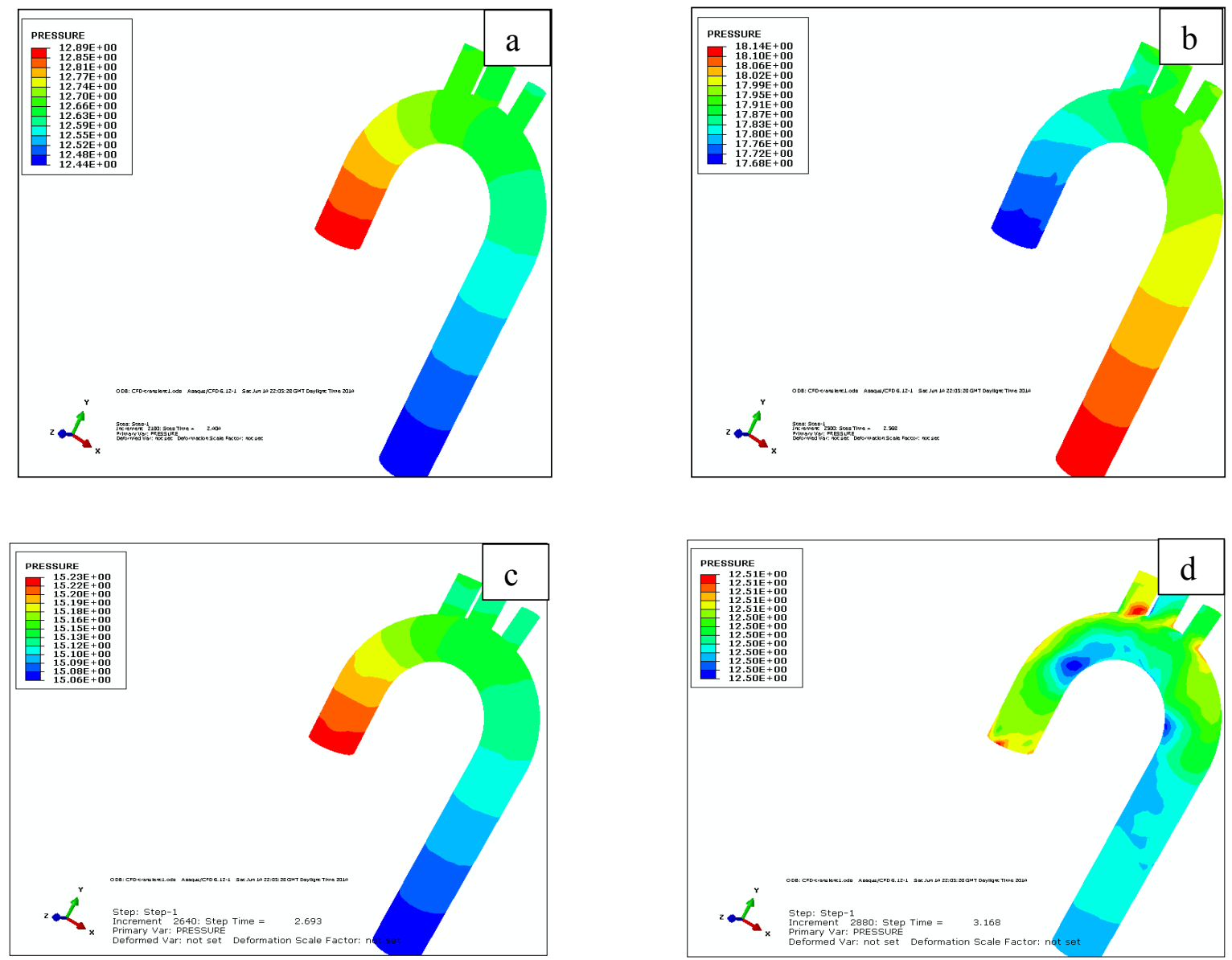

Fig. 7. The pressure distributions at (a) $t 1=2.404 \mathrm{~s}$, (b) $\mathrm{t} 2=2.568 \mathrm{~s}$, (c) $\mathrm{t} 3=2.693 \mathrm{~s}$, (d) $\mathrm{t} 4=3.168 \mathrm{~s}$
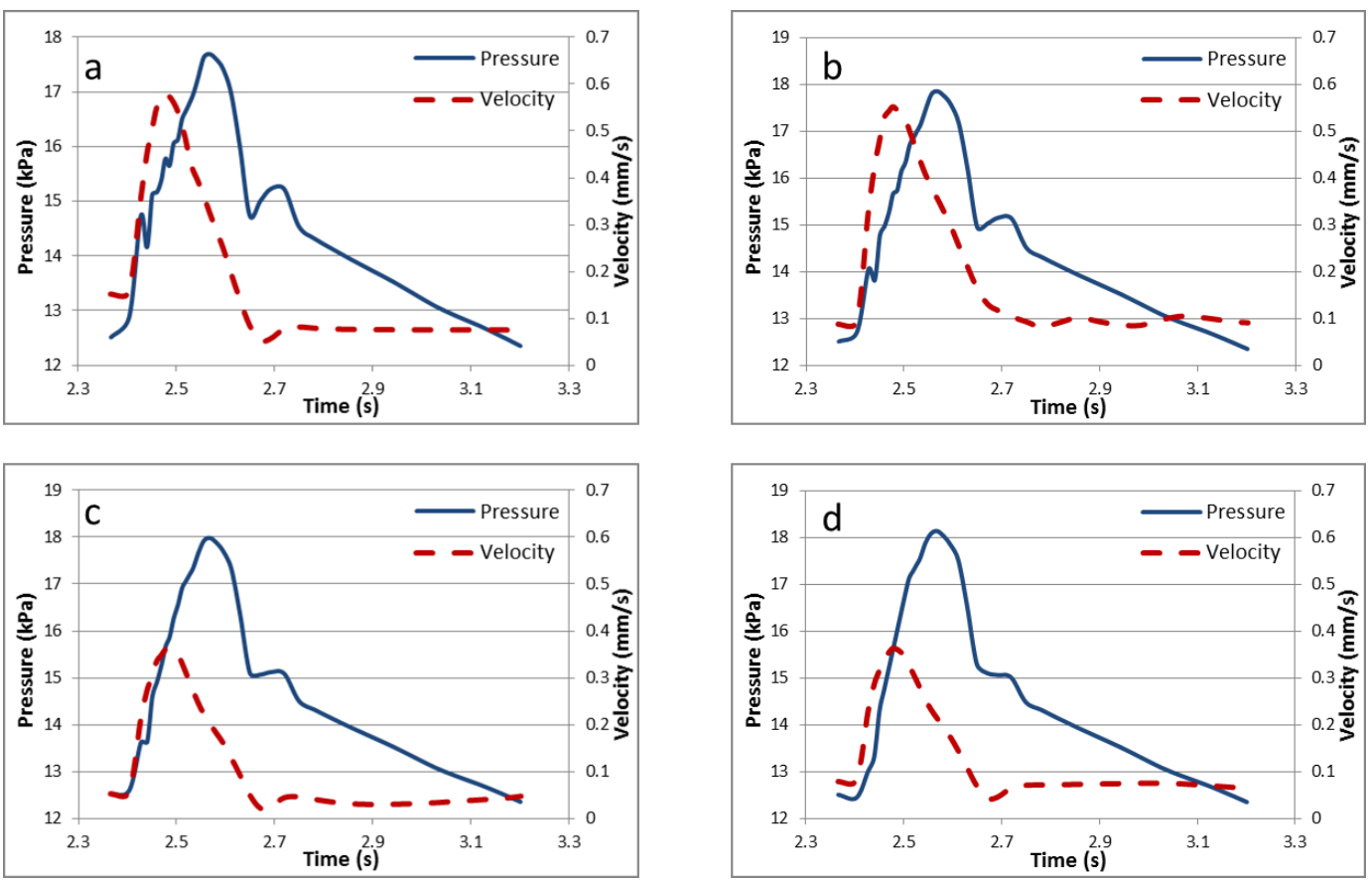

Fig. 8 Pressure and velocity waveforms at four locations: (a) inlet of the ascending aorta; (b) beginning of the aortic arch; (c) end of the aortic arch; (d) outlet of descending aorta. 


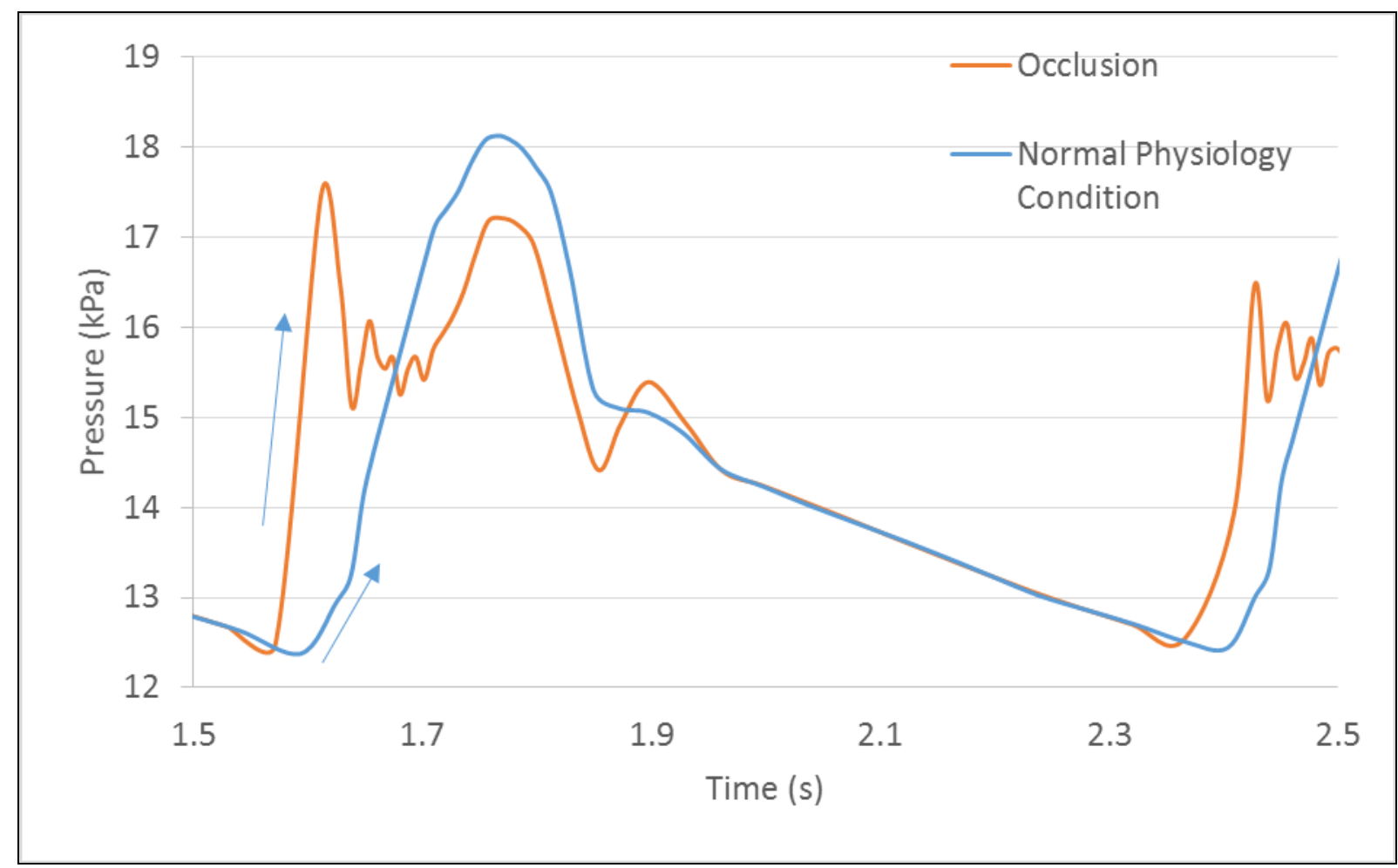

Fig. 9. Comparison of the pressure waveforms at the outlet of descending aorta between two conditions: the normal physiological conditions and when it is occluded due to the impact loading

Comparison between these two conditions indicated the wavefronts of pressure when the aorta is subjected to occlusion is approximately 3 times steeper than those in the normal physiological conditions. The peak of systolic pressure with the occlusion of descending aorta is slightly lower than that with the normal physiological conditions.

\section{Blood flow behaviour when the aorta is subjected to impact loading waveform}

If the peripheral arteries are experienced with the external impact loading, the additional pressure waveforms could be generated by the impact loading and propagates backwards to the heart. Patterns of blood flow propagation could be altered due to the additional wave generated by the impact loading and the reflected waves caused by the occlusions. In this study, it is assumed that the instant peak of pressure is generated at the descending aorta during the accident. The simulation results shown that the instant change of pressure fully altered the patters of the pressure and velocity waveforms, where the feature of cardiac blood flow cycle is fully lost. The peak of velocity under the impact loading is 1000 times more than those in the normal conditions, which could not be explained in this paper.

\section{Discussions and Conclusions}

In this paper, the occlusions and the pattern of blood wave propagations due to the impact are investigated using the following approaches. An aortic CFD (computational fluid dynamic) model representing the inside fluid domain of the aorta (aortic lumen) has been developed by using the ABAQUS CFD software to simulate the blood flow behaviour during car crash. The pattern of the pulsatile wave propagation in the healthy adult were investigated in three conditions: (1) normal physiological conditions; (2) the descending aorta was occluded; (3) descending aorta was subjected to the impact loading. 
The pressure and velocity waveforms at the varied locations are extracted from the CFD simulation results. The effect of occlusion and the impact loading upon the alteration of the patterns of the wave propagation was analysed. As was expected, steeper wave-front of the pressure waveform was observed when the aorta is subjected to occlusion and impact loading. This may be attributed to the superstition of backward reflected waves resulting from the occlusion of arteries. In addition, it was expected that the impact load could results in the significant increase of pressure amplitude, which was not observed. Under the impact loading, the features of physiological cardiac pressure and flow waveform were fully lost, the reason of which could be the occurrence of shock waves. The results implied that shock wave could be formed if the very severe impact loading takes place in the location closer to the heart and in the systolic period. In this study, blood vessel wall of the aorta is considered as rigid, resulting in the interaction between the deformation of blood vessel wall and blood flow behaviour being neglected. As is known, the arterial vessel is composed with the three layers, each of those experiences non-linear elastic performance under the cyclic loading. Ignoring the non-elastic properties of arterial wall could underestimate the performance of blood flow under the impact loading. Therefore, more realistic modelling through using visco- or hyper-elastic deformable elements in the future which may contribute to a more biofidelic understanding of the phenomenon.

\section{REFERENCES}

1. Bertrand, S., et al., 2008, Traumatic Rupture of Thoracic Aorta in Real-World Motor Vehicle Crashes, Traffic Injury Prevention, 9(2), 153-161.

2. Burkhart, H. M., et al., 2000, Fatal Blunt Aortic Injuries : a Review of 242 Autopsy Cases, The Journal of Trauma Injury, Infection and Critical Care, 50 (1), 113-115.

3. Brundage, S. I. et al., 1998, The epidemiology of thoracic injuries in pedestrians. J Trauma: Injury Infect, Crit Care, 45(6), 1010-1014.

4. McKay, M. P., 2006, National Highway Traffic Safety Administration (NHTSA) Notes. Seat Belt Use in 2005:

Demographic Results, Annals of Emergency Medcine, 47(4): 370-371; discussion 371-372.

5. Schulman, C.I., et al., 2007, Incidence and crash mechanisms of aortic injury during the past decade, Journal of Trauma, Injury, Infection and Critical Care, 62 (3), 664-667.

6. Sastry,P., Field,M. Cuerden,R., Richens, D., 2010, Low-impact scenarios may account for two-thirds of blunt traumatic aortic rupture, Emergence Medical Journal, 27(5),341-344.

7. Welsh, R., Morris, A., Hassan,A., Charlton,J., 2006, Crash characteristics and injury outcomes for older passenger car occupants, Transportation Research Part F, 9, 322-334.

8. Lundewall , J., 1964, The mechanics of traumatic rupture of the aorta. Acta Pathol Microbiol Scand, 62: 34-36.

9. Schleyer, F., 1963, On adventitial Haemorrhages of the thoracic blood vessels. J Forensic Med, 10, 3-5.

10. Kroell, C, Schneider D, Nahum, A, 1974, Impact tolerance and response of the human thorax II. 18th Stapp Car Crash Conference, Society of Automotive Engineers, 400 Commonwealth Drive, Warrendale, Pennsylvania 15096

11. Crass, J.R., et al. 1990, A proposed new mechanism of traumatic aortic rupture: the osseous pinch, Radiology, 176, 645-649.

12. Symbas, P.N., 1977, Fundamentals of Clinical Cardiology of 96 Great Vessels Injury. Am Heart J, 93, 518-522.

13. Voges, I. et al, 2012, Normal values of aortic dimensions, distensibility, and pulse wave velocigy in children and young adults: a cross-sectional study, Journal of Cardiovascular Magnetic Resonance, 14:77

\section{BIOGRAPHY}

Dr Jiling Feng obtained her PhD degree from Brunel University in 2008. Dr Feng joined Manchester Metropolitan University as a Lecturer in Mechanical Engineering in 2012. She was also worked as a Research Fellow and Research Associate in University of Strathclyde and Loughborough University, respectively. Currently, Dr Feng focuses on her research in the areas of cardiovascular mechanics, invitro/in-vivo study for the mechanism of atherosclerosis in carotid arteries and coronary arteries. Her previous research experience includes ageing driver model and multiscaled FE modelling for the material damage performance.

Address:

Dr Jiling Feng, E347 John Dalton Building, Chester Street, Manchester, M1 5GD, UK

Tel: (+44) 1612471650

Email: j.feng@mmu.ac.uk 